\title{
Uso de tartarato de brimonidina para resolução de matting telangiectásico: relato de caso
}

\author{
Use of brimonidine tartrate to resolve telangiectatic matting: case report
}

Brenno Augusto Seabra de Mello Netto ${ }^{1}$ (D), Yasmin de Rezende Beiriz ${ }^{2}$ (D), Américo Carnelli Bonatto ${ }^{3}$ (D), Gustavo Sasso Benso Maciel' (D), Laila Reggiani de Almeida (D) José Marcelo Corassa ${ }^{1}$

\begin{abstract}
Resumo
A escleroterapia é, atualmente, o tratamento de escolha para telangiectasias e veias reticulares, apresentando nível de recomendação $1^{\text {a }}$ pela diretriz europeia para escleroterapia. Os efeitos colaterais mais comuns desse procedimento são a hiperpigmentação e o matting telangiectásico, sendo este último um dos mais temidos em virtude do dano estético e da dificuldade de tratamento. O matting se refere a vasos com diâmetro inferior a 0,2 mm que podem surgir esporadicamente ou em áreas bem definidas, principalmente nos membros inferiores. Este relato apresenta um caso de matting tratado com o uso de tartarato de brimonidina tópico.
\end{abstract}

Palavras-chave: escleroterapia; telangiectasia; varizes; relato de caso.

\begin{abstract}
Sclerotherapy is currently the treatment of choice for telangiectasias and reticular veins, with grade $1^{\mathrm{a}}$ recommendation in the European Guideline for sclerotherapy. The most common side effects of this procedure are hyperpigmentation and telangiectatic matting, the second of which provokes great concern because of the esthetic damage and the difficulty of treatment. Matting refers to vessels with a diameter of less than $0.2 \mathrm{~mm}$, which may emerge irregularly or in well-defined areas, especially on the lower limbs. This report presents a case of matting treated with topical Brimonidine Tartrate.
\end{abstract}

Keywords: sclerotherapy; telangiectasis; varicose veins; case report.

Como citar: Mello Netto BAS, Beiriz YR, Bonatto AC, Maciel GSB, Almeida LR, Corassa JM. Uso de tartarato de brimonidina para resolução de matting telangiectásico: relato de caso. J Vasc Bras. 2020;19: e20190159. https://doi. org/10.1590/1677-5449.190159

${ }^{1}$ Venno Clinic - Excelência Vascular, Departamento de Cirurgia Vascular, Vitória, ES, Brasil.

${ }^{2}$ Escola Superior de Ciências da Santa Casa de Misericórdia de Vitória - EMESCAM, Curso de Graduação em Medicina, Vitória, ES, Brasil.

${ }^{3}$ Faculdade Brasileira MULTIVIX, Curso de Graduação em Medicina, Vitória, ES, Brasil.

Fonte de financiamento: Nenhuma.

Conflito de interesse: Os autores declararam não haver conflitos de interesse que precisam ser informados.

Submetido em: Janeiro 16, 2020. Aceito em: Maio 01, 2020 


\section{INTRODUÇÃO}

A classificação das doenças venosas é baseada em dados clínicos $(\mathrm{C}$, etiologia (E), distribuição anatômica (A) e fisiopatologia (P), denominada classificação $\mathrm{CEAP}^{1}$. As telangiectasias e as veias reticulares compreendem a classe 1 (C1) da classificação clínica de $\mathrm{CEAP}^{2}$, e o tratamento de escolha para as telangiectasias é a escleroterapia ${ }^{3}$.

Atualmente, os esclerosantes mais utilizados são polidocanol e glicose hipertônica ${ }^{4}$. Contudo, cada tratamento apresenta uma série de complicações, sendo os efeitos colaterais mais comuns da escleroterapia a hiperpigmentação e o matting ${ }^{4}$.

Nesse contexto, o matting telangiectásico, também conhecido como nuvem telangiectásica, é um dos mais temidos pelo dano estético e pela dificuldade de tratamento. $\mathrm{O}$ matting se refere a vasos com diâmetro inferior a $0,2 \mathrm{~mm}$ que podem surgir esporadicamente ou em áreas bem definidas, principalmente nos membros inferiores ${ }^{5}$. A angiogênese e a vasodilatação são fatores associados ao matting, ainda que não se tenha uma causa plenamente estabelecida ${ }^{6}$. Assim, algumas hipóteses foram criadas, como a do estrogênio e a das reações inflamatórias locais.

Sabe-se que as células endoteliais apresentam receptores de estrogênio, o que sugere que o estrogênio endógeno e exógeno apresenta um papel na angiogênese, favorecendo o aparecimento de matting. Além disso, fatores inflamatórios como a fibronectina podem atacar a membrana basal do endotélio, podendo levar à angiogênese ${ }^{7}$.

O relato a seguir apresenta um caso de matting macroscopicamente resolvido com o uso de tartarato de brimonidina tópico.

\section{DESCRIÇÃO DO CASO}

Uma paciente feminina de 19 anos, Fitzpatrick II, não tabagista, sedentária, sem comorbidades ou alergia medicamentosa e em uso regular de anticoncepcional oral apresentava queixa estética motivada por telangiectasias combinadas na face lateral da coxa esquerda. Foi orientada quanto à necessidade de hidratação cutânea e uso de picnogenol $200 \mathrm{mg} /$ dia por via oral por pelo menos 15 dias antes de passar por procedimento. Foi realizada fotodocumentação detalhada, e a paciente recebeu o termo de consentimento com informações detalhadas do tratamento e possíveis complicações. Ao retornar após 15 dias, a paciente foi submetida a escleroterapia convencional em telangiectasias combinadas na face lateral da coxa esquerda, com solução de glicose $65 \%$ e polidocanol $0,5 \%$ (volume total de $1,5 \mathrm{~mL}$ ).
$\mathrm{Na}$ área que margeava os vasos de interesse, desenvolveu nuvem telangiectásica de grandes dimensões (10 x $15 \mathrm{~cm}$ aproximadamente) cerca de 2 dias após o procedimento (Figura 1). No exame de realidade aumentada e de eco-Doppler venoso da região, não foram visualizadas varizes ou veias nutridoras que poderiam ter associação com a complicação em questão.

A conduta inicial contemplava, além de tranquilizar a paciente, manter a não exposição solar e o uso de picnogenol oral e tópico. Foram realizadas, ainda, três sessões de luz intensa pulsada $540 \mathrm{~nm} / 17 \mathrm{~J} / 15 \mathrm{~ms}$ (parâmetros similares ao tratamento de rosácea), com intervalo de 21 dias entre as sessões. Aos olhos do mesmo avaliador e da paciente, houve apenas leve atenuação do aspecto (Figura 2).

Portanto, foi proposto o uso de tartarato de brimonidina $0,5 \%$ por via tópica com uma aplicação diária. Após 7 dias do uso regular da medicação, houve melhora considerável da lesão (Figura 3). Optamos em conjunto

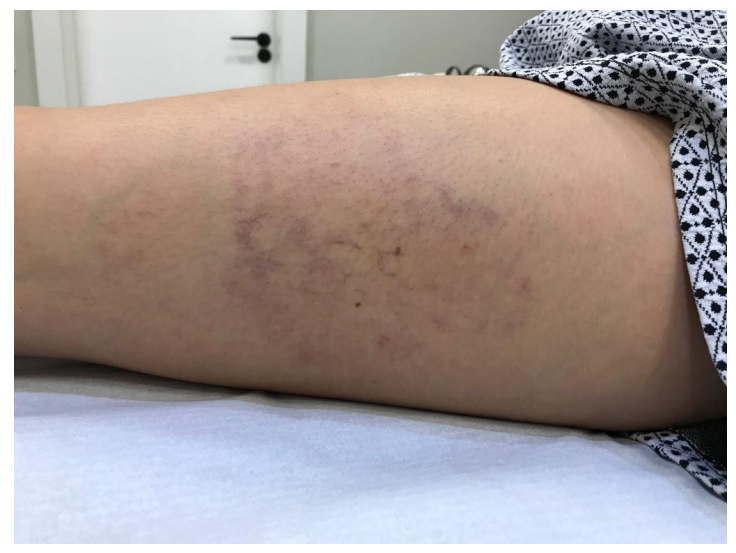

Figura 1. Dois dias após escleroterapia, com surgimento de matting telangiectásico.

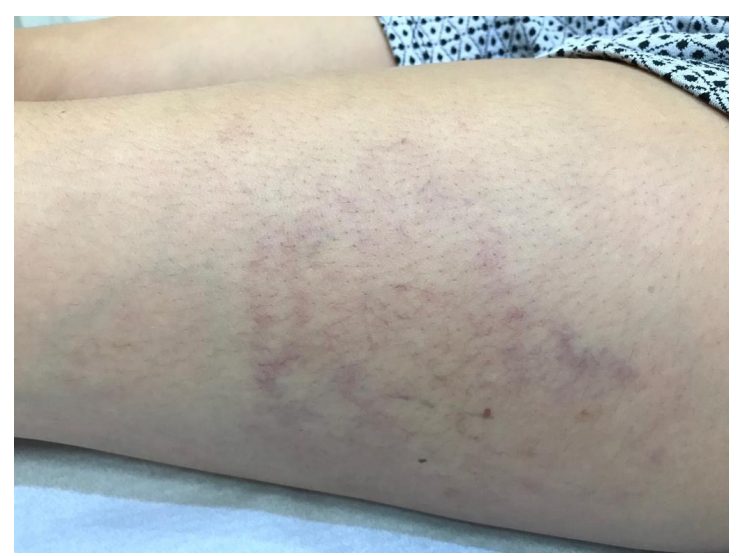

Figura 2. Resultado insatisfatório após tentativa de tratamento com luz intensa pulsada. 
por continuar por mais 7 dias, com resultado muito satisfatório (Figura 4). Após 14 dias, suspendemos o uso da medicação e seguimos acompanhando apenas clinicamente a paciente, que se encontra satisfeita,

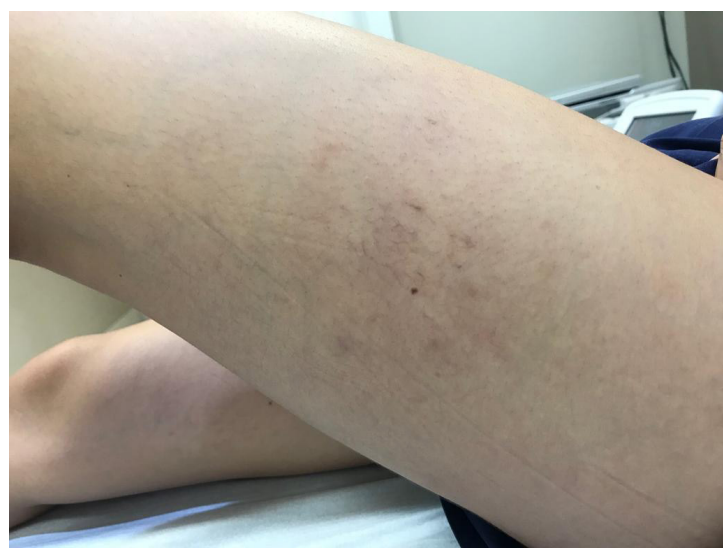

Figura 3. Aspecto da lesão após 1 semana do uso de tartarato de brimonidina.

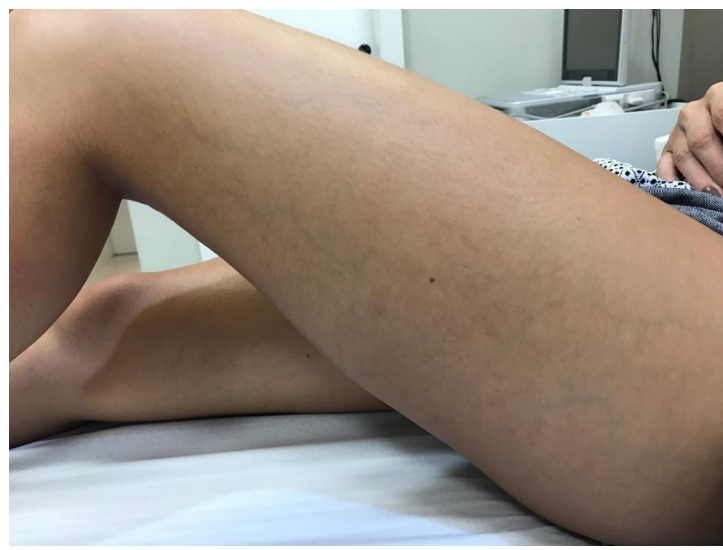

Figura 4. Resultado após 2 semanas do uso do tartarato de brimonidina.

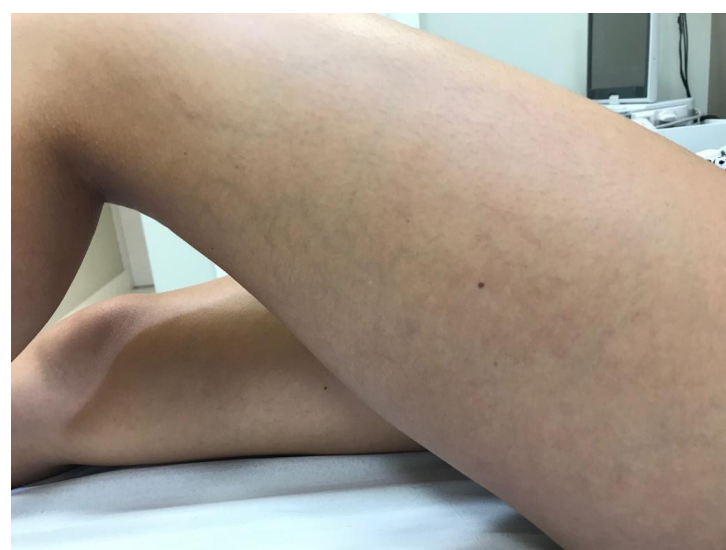

Figura 5. Acompanhamento em 6 meses após aparecimento do matting telangiectásico. com completo desaparecimento das telangiectasias e do matting telangiectásico. A iniciativa de tratamento com tartarato de brimonidina foi baseada no uso do Mirvaso ${ }$, medicamento utilizado para rosácea cuja fisiopatologia possui algumas similaridades com o matting. Em virtude da dificuldade de encontrar esse medicamento, optou-se pela manipulação da medicação. A paciente encontra-se em seguimento clínico há 6 meses (Figura 5), e não foi observado efeito rebote até o momento.

\section{DISCUSSÃO}

A etiologia do matting telangiectásico é desconhecida, mas sabe-se que ocorre mais em mulheres e apresenta, como fatores de risco, história familiar de telangiectasia, excesso de hormônios femininos exógenos e obesidade ${ }^{8}$. O matting pós-escleroterapia ocorre em aproximadamente 15 a 20\% dos tratamentos e consiste no aparecimento de pequenas telangiectasias vermelhas na área da veia tratada ${ }^{4}$. Ele se caracteriza pelo surgimento de uma pigmentação irregular de início em 4 a 6 semanas após o tratamento. Utilizar concentração mínima de esclerosante, pequenos volumes e baixa pressão durante a escleroterapia são medidas técnicas para evitar essa complicação ${ }^{4}$. Outrossim, classificar o fototipo cutâneo segundo Fitzpatrick e fazer hidratação com picnogenol préprocedimento são cuidados que podem promover melhores desfechos.

$\mathrm{O}$ matting pode ser transitório (com resolução espontânea de 3 a 12 meses após o tratamento) ou permanente 9 . O tratamento inicial é baseado em localizar o refluxo proximal não tratado de veias safenas, perfurantes, tributárias ou reticulares ${ }^{10}$. Investigar comorbidades associadas é de grande importância para definição da etiologia da reação, uma vez que se observou maior tendência ao desenvolvimento de matting nos pacientes alérgicos, com distúrbio de sangramento, diagnóstico de asma brônquica ou terapia hormonal em curso ${ }^{5}$.

Um olhar atento sobre a escolha do esclerosante, cuidado na técnica de punção e seleção dos vasos de interesse, bem como sessões com menor volume e pressão, podem driblar o resultado negativo. No cenário atual da flebologia, não se conhece um tratamento por via tópica eficaz para as alterações vasomotoras do matting. A relevância clínica deste relato de caso está principalmente relacionada a um tratamento tópico alternativo para o tratamento do matting. As opções de laser como luz intensa pulsada ou Nd YAG 1064 vêm para complementar o arsenal terapêutico e têm seu espaço nos casos de pacientes com fobia de punção ou em adjuvância ${ }^{11}$. 
Sabe-se que a potência da solução utilizada para escleroterapia pode ser um fator causador do matting. O uso de glicose hipertônica isolada poderia, talvez, ser um fator para evitar a complicação apresentada. Todavia, este relato de caso objetiva analisar uma possibilidade de tratamento da complicação que, no caso, é o matting telangiectásico.

Diante desse cenário, o tartarato de brimonidina, usualmente utilizado para rosácea, surge como um tratamento alternativo para o matting telangiectásico. Esse medicamento é um agonista alfa-2 adrenérgico seletivo $^{12}$. Seu uso tópico se mostrou seguro e com adequada tolerabilidade, não apresentando efeitos colaterais de grande magnitude ${ }^{12}$.

Erin Lowe ${ }^{13}$ descreveu o aparecimento de eritema paradoxal reacional com uso prolongado nos casos de rosácea, o que foi resolvido com descontinuação do tratamento por 48 horas, com completa resolução do quadro de rash. Explicações detalhadas sobre os riscos do procedimento, refinamento estético, clareza, conhecimento sobre os tratamentos e boa relação médico-paciente são indispensáveis para a adesão do paciente a um tratamento difícil e, às vezes, oneroso.

O uso de tartarato de brimonidina no caso de matting descrito trouxe resolução para a queixa da paciente, sem apresentar efeitos colaterais. Uma opção de tratamento tópico para telangiectasia secundária pode acrescentar novas perspectivas para a flebologia. Embora essa medicação possa ser uma nova opção para o tratamento do matting telangiectásico, estudos clínicos prospectivos com amostra calculada devem ser realizados para que se possa obter maior evidência científica dessa indicação de uso, além de averiguar a segurança do tratamento para essa condição específica.

\section{REFERÊNCIAS}

1. França LH, Tavares V. Insuficiência venosa crônica: uma atualização. J Vasc Bras. 2003;2(4):18-28.

2. Castro e Silva M, Cabral ALS, Barros N Jr, Castro AA, Santos MERC. Diagnóstico e tratamento da doença venosa crônica. J Vasc Bras. 2005;4(3):S185-94.

3. Jia X, Mowatt G, Burr JM, Cassar K, Cook J, Fraser C. Systematic review of foam sclerotherapy for varicose veins. Br J Surg. 2007;94(8):92536. http://dx.doi.org/10.1002/bjs.5891. PMid:17636511.

4. Yiannakopoulou E. Safety concerns for sclerotherapy of telangiectases, reticular and varicose veins. Pharmacology. 2016;98(1-2):62-9. http://dx.doi.org/10.1159/000445436. PMid:27104778.

5. Kadam P, Lim J, Paver I, Connor DE, Parsi K. Telangiectatic matting is associated with hypersensitivity and a bleeding tendency. Eur J Vasc Endovasc Surg. 2018;55(4):554-9. http://dx.doi.org/10.1016/j. ejvs.2017.12.013. PMid:29409702.

6. Goldman MP, Martin DE, Fitzpatrick RE, Ruiz-Esparza J. Pulsed dye laser treatment of telangiectases with and without subtherapeutic sclerotherapy. J Am Acad Dermatol. 1990;23(1):23-30. http:// dx.doi.org/10.1016/0190-9622(90)70180-P. PMid:2365873.

7. Sadick NS, Urmacher C. Estrogen and progesterone receptors: their role in postsclerotherapy angiogenesis telangiectatic matting (TM). Dermatol Surg. 1999;25:7. http://dx.doi.org/10.1046/j.15244725.1999.99026.x. PMID: 10469110.

8. Davis LT, Duffy DM. Determination of incidence and risk factors for postsclerotherapy telangiectatic matting of the lower extremity: a retrospective analysis. J Dermatol Surg Oncol. 1990;16(4):327-30. http://dx.doi.org/10.1111/j.1524-4725.1990.tb00043.x. PMid:1691217.

9. Goldman MP, Sadick NS, Weiss RA. Cutaneous necrosis, telangiectatic matting, and hyperpigmentation following sclerotherapy. Etiology, prevention, and treatment. Dermatol Surg. 1995;21(1):19-29, quiz 31-2. http://dx.doi.org/10.1111/j.1524-4725.1995.tb00107.x. PMid:7600016.

10. Mann MW. Sclerotherapy: it is back and better. Clin Plast Surg. 2011;38(3):475-87, vii. http://dx.doi.org/10.1016/j.cps.2011.02.006. PMid:21824544.

11. Parlar B, Blazek C, Cazzaniga S, et al. Treatment of lower extremity telangiectasias in women by foam sclerotherapy vs. Nd:YAG laser: a prospective, comparative, randomized, open-label trial. J Eur Acad Dermatol Venereol. 2015;29(3):549-54. http://dx.doi. org/10.1111/jdv.12627. PMid:25069999.

12. Del Rosso JQ. Management of facial erythema of rosacea: what is the role of topical a-adrenergic receptor agonist therapy. J Am Acad Dermatol. 2013;69(6, Suppl 1):44-56. http://dx.doi.org/10.1016/j. jaad.2013.06.009. PMid:24229637.

13. Lowe E, Lim S. Paradoxical erythema reaction of long-term topical brimonidine gel for the treatment of facial erythema of rosacea. J Drugs Dermatol. 2016;15(6):763-5. PMid:27272086.

Correspondência Brenno Augusto Seabra de Mello Netto Venno Clinic - Excelência Vascular, Departamento de Cirurgia Vascular Rua Aleixo Neto, 807/802, Ed Ilha de Vitória - Praia do Canto CEP 29055-145 - Vitória (ES), Brasil Tel.: (27) 99223-3757 E-mail:vennoclinic@gmail.com

Informações sobre os autores BASMN, GSBM e LRA - Cirurgiões vasculares, Universidade Federal do Espírito Santo (UFES). YRB - Estudante de Medicina, Escola Superior de Ciências da Santa Casa de Misericórdia de Vitória (EMESCAM). ACB - Estudante de Medicina, Faculdade Brasileira MULTIVIX (MULTIVIX).

JMC - Cirurgião vascular, Hospital da Lagoa.

Contribuições dos autores Concepção e desenho do estudo: BASMN, YRB, ACB, LRA, GSBM, JMC Análise e interpretação dos dados: BASMN, YRB, ACB, LRA, GSBM, JMC Coleta de dados: BASMN, YRB, ACB, LRA, GSBM, JMC Redação do artigo: BASMN, YRB, ACB, LRA, GSBM, JMC Revisão crítica do texto: BASMN, YRB, ACB, LRA, GSBM, JMC Aprovação final do artigo*: BASMN, YRB, ACB, LRA, GSBM, JMC Análise estatística: BASMN, YRB, ACB, LRA, GSBM, JMC Responsabilidade geral pelo estudo: BASMN

*Todos os autores leram e aprovaram a versão final submetida ao J Vasc Bras. 